\title{
La coautoría como expresión de la colaboración en la producción científica de Camagüey
}

\author{
Dianelis Olivera Batista \\ Universidad de Camagüey "Ignacio Agramonte Loynaz" - Camagüey, Cuba \\ María Josefa Peralta González \\ Universidad Central "Marta Abreu" de Las Villas - Santa Clara, Cuba \\ Ognara García García \\ Universidad de Camagüey "Ignacio Agramonte Loynaz" - Camagüey, Cuba
}

ORIGINAL

\section{Resumo}

Objetivo: Describir el comportamiento de la coautoría en la producción científica de Camagüey registrada en las bases de datos de Web of Science (WoS) y Scopus en el período 2001-2013.

Método: Se realizó un análisis bibliométrico mediante la aplicación de indicadores tales como índice de coautoría, rango de autores firmantes y su relación con las citaciones recibidas, evolución de la colaboración; se utilizó el análisis de redes para el estudio y visualización de la colaboración.

Resultados: El índice de coautoría de la producción científica en general es 4.85 y el rango de autores firmantes predominante se encuentra entre 4 y 5 autores. El artículo con mayor cantidad de autores (30) es el que mayor cantidad de citas ha recibido en las dos bases de datos analizadas. Sólo 50 documentos no muestran colaboración al ser firmados por un único autor, mientras que la colaboración intrainstitucional está presente en más de 200 documentos.

Conclusiones: Los patrones de colaboración muestran el predominio de la colaboración intrainstitucional en el período. En el análisis de la colaboración institucional se destacan la Universidad de Camagüey y el Hospital Manuel Ascunce Domenech al establecer vínculos colaborativos más fuertes con otras entidades, tanto cubanas como extranjeras. La internacionalización de la producción científica fue muy baja hasta el año 2005, a partir del 2006 tiene un ligero incremento pero continúa por debajo de la colaboración nacional e intrainstitucional.

Palavras-chave

Camagüey; coautoría; colaboración científica; producción científica; Scopus; Web of Science

\section{Co-authorship as expression of collaboration in the scientific production of Camagüey}

\section{Abstract}

Objective: To describe the behavior of co-authorship in the scientific production of Camagüey registered in the databases of Web of Science (WoS) and Scopus in the period 2001-2013.

Method: A bibliometric analysis was performed through the application of indicators such as co-authorship index, rank of authors and their relation with citations received, evolution of collaboration; the network analysis was used for the study and visualization of the collaboration.

Results: The co-authorship index of scientific production in general is 4.85 and the rank of predominant authors is between 4 and 5 authors. The article with the greatest number of authors (30) is the one with the highest number of citations received in the two databases analyzed. Only 50 documents do not show collaboration because were signed by a single author, while intra-institutional collaboration is present in more than 200 documents.

Conclusions: The patterns of collaboration show the predominance of intrainstitutional collaboration in the period. In the analysis of institutional collaboration, the University of Camagüey and the Manuel Ascunce Domenech Hospital stand out by establishing stronger collaborative links with other entities, both Cuban and foreign. The internationalization of scientific production was very low until 2005, as of 2006 it has a slight increase but continues below the national and intrainstitutional collaboration. 


\section{Introducción}

La coautoría es una de las formas más tangibles y bien documentadas de colaboración científica (Glänzel y Schubert, 2004). Los científicos colaboran para ganar visibilidad, reputación, habilidades complementarias y acceso a recursos. Estos factores se relacionan con la organización social e intelectual de la comunidad científica, y no con factores históricos, de proximidad geográfica y el modelo económico centro-periferia (Leydesdorff y Wagner (2009).

Russell y otros (2009) defienden que, a pesar de las limitaciones e imperfecciones del método, a través del análisis de trabajos publicados en coautoría, es posible llegar a una representación adecuada, mientras no necesariamente precisa, de las colaboraciones científicas; a mayor nivel de abstracción, mayor confianza en esta aproximación.

La coautoría aumenta la visibilidad de los resultados debido a que están expuestos a un mayor número de investigadores, gracias al mayor número de contactos personales (Franceschet y Costantini, 2010). Los artículos con varios autores tienen mayor mérito científico y, a su vez, contienen menor cantidad de errores debido a las contribuciones de un equipo multidisciplinar (Padial y otros, 2010).

Los estudios bibliométricos, en muchos casos, analizan la coautoría y su relación con el impacto de las publicaciones. Diversas investigaciones han descubierto una correlación positiva entre el número de instituciones firmantes de artículos y el impacto científico (Glänzel, 2002), (Vieira y Gomes, 2010), (Gazni y Didegah, 2011).

Desde mediados del siglo XX la producción del conocimiento se realiza cada vez más en el seno de una amplia y heterogénea red de investigadores que trabajan de forma cooperativa organizados en grupos de investigación cuyos miembros pueden estar adscritos a instituciones o países diferentes, e incluso pueden no conocerse personalmente y comunicarse por teléfono o a través de Internet (González-Alcaide y Gómez, 2014).

Al considerar la colaboración científica internacional como un sistema auto-organizado con características de redes, las interacciones entre los científicos se tornan importantes, pues revelan algunas dinámicas (Souza y Chittó, 2010). La utilización complementaria de las técnicas bibliométricas y el análisis de redes se ha develado como una combinación mucho más útil de lo que se podría esperar de la simple suma de dos métodos distintos. Los gráficos de redes permiten objetivar grupos de trabajo que de otro modo sería difícil descubrir (Osca-Lluch, 2010).

Varios estudios bibliométricos, analizan la coautoría desde una perspectiva institucional (Russell y otros, 2006), (Olmeda-Gómez y otros, 2008), (Gazni y Didegah, 2011). Otras investigaciones han analizado la coautoría en un espacio geográfico, donde se interrelacionan instituciones con distintas características, un ejemplo de este tipo de estudio es el de (Sancho, 2006): Indicadores de colaboración científica inter-centros en los países de América Latina.

El presente trabajo tiene como objetivo describir el comportamiento de la coautoría en la producción científica de Camagüey registrada en las bases de datos de Web of Science (WoS) y Scopus en el período 2001-2013, mediante la aplicación de indicadores bibliométricos y el análisis de redes.

\section{Metodología}

Para realizar el estudio se utilizaron las bases de datos Science Citation Index, Social Science Citation Index, y Arts and Humanities Citation Index, pertenecientes a WoS, y la base de datos Scopus como fuentes de información. Las mismas ofrecen información sobre la institución de adscripción de los autores, lo que las hace factibles para estudiar la coautoría. Además, se tuvo en cuenta el hecho de que la producción científica representada en las mismas constituye la más visible a nivel internacional.

Se tuvieron en cuenta ambas bases de datos, dado que investigaciones como la de (Gavel e Iselid, 2008) demuestran que existen niveles de solapamiento entre las dos, pero también existen diferencias, por ejemplo: mientras que Scopus contiene 6256 revistas que no cubre WoS, ésta última cuenta con 1467 revistas no recogidas por Scopus. La mayor cantidad de publicaciones que indexa Scopus amplía la cobertura idiomática y geográfica. 
Con el objetivo de recuperar todos los registros con al menos un autor de la provincia Camagüey se efectuó la pesquisa en el campo de Author Address en ambas bases de datos y como resultado se recuperaron 213 registros en WoS y 584 en Scopus. Se determinó la existencia de 173 registros que coincidían en ambas bases de datos, 40 publicaciones registradas en WoS que no están en Scopus, y 411 que están en Scopus y no se encuentran en WoS. Para el estudio de la colaboración se decidió analizar una sola vez las publicaciones que aparecían en ambas bases de datos, por tanto, se trabajó con un total de 624 registros.

Se llevó a cabo la normalización de los campos Author, Author Address, además se trabajó con el campo Notes para el análisis de las citas recibidas. En el caso de este último se analizaron por separado los 213 registros recuperados en WoS y los 584 recuperados en Scopus, debido a que la cantidad de citas recibidas por las publicaciones en cada base de datos es diferente.

El cálculo de los indicadores se realizó mediante Microsoft Excel, del paquete de programas Microsoft Office, y también Bibexcel. Este último se utilizó para la obtención de matrices, cuyo procesamiento mediante Ucinet $v 6.0$ permitió la representación visual de las redes de colaboración y el análisis de estas. El análisis de redes sociales permite el cálculo de medidas de centralidad, las cuales permiten establecer la posición e importancia de un actor dentro de la red, así como determinar el papel que este desempeña (Delgado et al., 2006). El grado de centralidad expresa el porcentaje de lazos que tiene un actor, cuanto mayor es el grado, mayor será el número de personas con las que se conecta un nodo.

Los indicadores analizados fueron: productividad por sectores, índice de coautoría, rango de autores firmantes, porciento de documentos citados, promedio de citas por documentos. En el estudio de los indicadores de colaboración se consideraron 5 categorías:

- Documentos sin colaboración: publicaciones firmadas por un solo autor.

- Documentos en colaboración intrainstitucional: publicaciones firmadas por más de un autor de una misma institución camagüeyana.

- Documentos en colaboración nacional: publicaciones firmadas en conjunto con uno o más investigadores de otras instituciones cubanas. Dentro de esta se valora como colaboración territorial las publicaciones firmadas por autores de diferentes instituciones camagüeyanas.

- Documentos en colaboración internacional: publicaciones donde aparece al menos la firma de un autor extranjero.

- Documentos en colaboración internacional-nacional: publicaciones donde aparece al menos la firma de un autor extranjero unido a la firma de uno o más investigadores de entidades nacionales.

\section{Resultados}

En las publicaciones analizadas la filiación de los autores camagüeyanos indica que proceden de instituciones pertenecientes a diferentes sectores científicos o económicos. La mayor producción científica se concentra en el sector de la salud, seguido por la Universidad de Camagüey, instituciones pertenecientes al Ministerio de Ciencia, Tecnología y Medio Ambiente (CITMA), y el Centro de Ingeniería Genética y Biotecnología (CIGB) (figura 1).

El hecho de que el sector de la salud sea el más productivo está dado por la unificación de las contribuciones de un gran número de instituciones (Universidad de Ciencias Médicas, Centro de higiene y epidemiología, Centro de promoción y educación para la salud, varios hospitales, policlínicos, clínicas estomatológicas, y otras entidades), mientras que la Universidad de Camagüey es una única institución al igual que el CIGB, y el CITMA sólo agrupa 3 organizaciones (Centro Meteorológico Provincial, Centro de Investigaciones de Medio Ambiente, y Centro de Ingeniería Ambiental). 
Figura 1. Producción científica camagüeyana por sectores en Scopus y WoS (2001-2013).

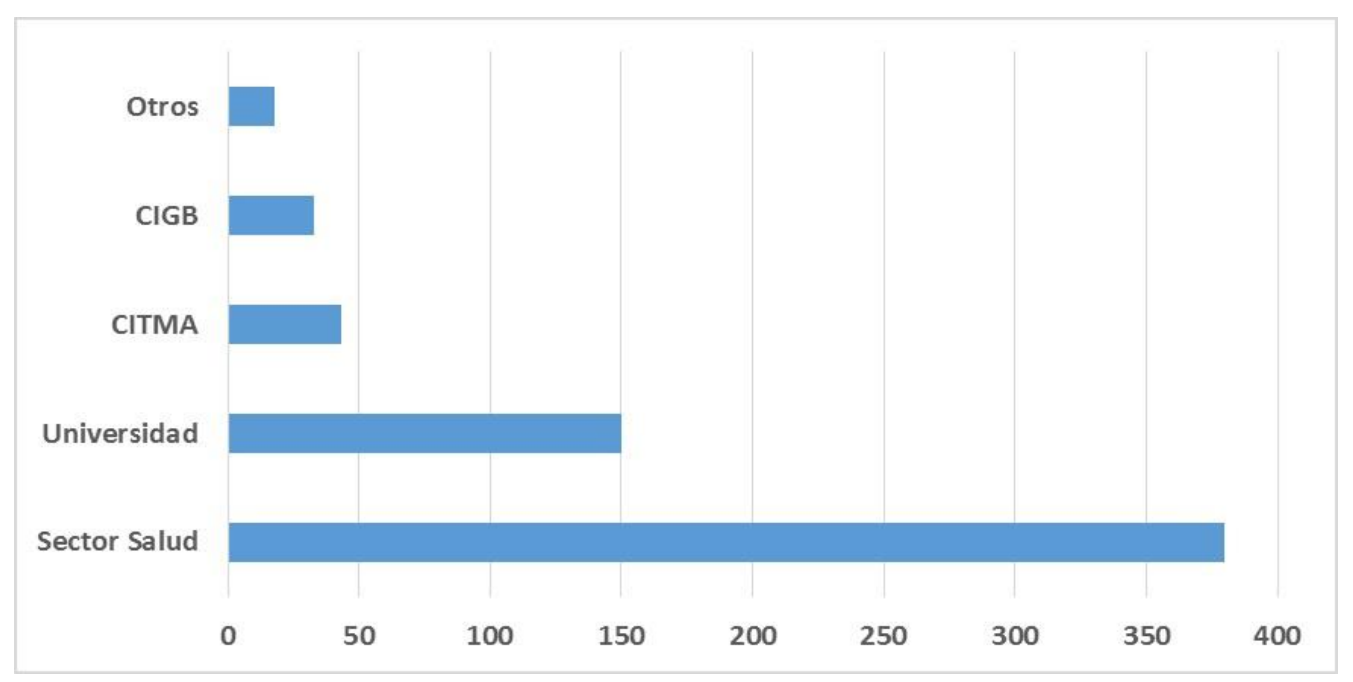

Fuente: Elaboración propia.

No obstante, sin importar la institución o sector de procedencia una característica predominante en la producción científica camagüeyana es la coautoría. Sólo el $8 \%$ de la producción total fue originada por autores individuales. El índice de coautoría es diferente en cada una de las bases de datos analizadas, mientras que en Scopus su valor es 4,61 (tabla 1) en WoS es 6,06 (tabla 2). Esto se debe a las diferencias entre la cantidad de trabajos registrados en cada una de estas fuentes, así como los desiguales conjuntos de firmas.

Tabla 1. Cantidad de firmas, número de trabajos, y cálculo del índice de coautoría en Scopus.

\begin{tabular}{|c|c|c|c|c|c|c|}
\hline Firmas & Trabajos & $\%$ & \% Acum. & Firmas & $\%$ & \% Acum. \\
\hline 1 & 47 & 8,05 & 8,05 & 47 & 1,74 & 1,74 \\
\hline 2 & 70 & 11,99 & 20,04 & 140 & 5,2 & 6,94 \\
\hline 3 & 88 & 15,07 & 35,11 & 264 & 9,8 & 16,74 \\
\hline 4 & 123 & 21,07 & 56,18 & 492 & 18,27 & 35,01 \\
\hline 5 & 115 & 19,69 & 75,87 & 575 & 21,36 & 56,37 \\
\hline 6 & 55 & 9,42 & 85,29 & 330 & 12,26 & 68,63 \\
\hline 7 & 32 & 5,48 & 90,77 & 224 & 8,32 & 76,95 \\
\hline 8 & 15 & 2,57 & 93,34 & 120 & 4,46 & 81,41 \\
\hline 9 & 10 & 1,71 & 95,05 & 90 & 3,34 & 84,75 \\
\hline 10 & 5 & 0,86 & 95,91 & 50 & 1,86 & 86,61 \\
\hline 11 & 4 & 0,68 & 93,59 & 44 & 1,63 & 88,24 \\
\hline 12 & 6 & 1,03 & 97,62 & 72 & 2,68 & 90,92 \\
\hline 13 & 2 & 0,34 & 97,96 & 26 & 0,96 & 91,88 \\
\hline 14 & 1 & 0,17 & 98,13 & 14 & 0,52 & 92,4 \\
\hline 15 & 4 & 0,68 & 98,81 & 60 & 2,22 & 94,62 \\
\hline 16 & 2 & 0,34 & 99,15 & 32 & 1,19 & 95,81 \\
\hline 17 & 1 & 0,17 & 99,32 & 17 & 0,63 & 96,44 \\
\hline 18 & 1 & 0,17 & 99,49 & 18 & 0,67 & 97,11 \\
\hline 22 & 1 & 0,17 & 99,66 & 22 & 0,82 & 97,93 \\
\hline 26 & 1 & 0,17 & 99,83 & 26 & 0,96 & 98,89 \\
\hline \multirow[t]{3}{*}{30} & 1 & 0,17 & 100 & 30 & 1,11 & 100 \\
\hline & 584 & & & 2693 & & \\
\hline & & & Índice: 4,61 & & & \\
\hline
\end{tabular}

Fuente: Elaboración propia. 
Tabla 2. Cantidad de firmas, número de trabajos, y cálculo del índice de coautoría en WoS.

\begin{tabular}{|c|c|c|c|c|c|c|}
\hline Firmas & Trabajos & $\boldsymbol{\%}$ & $\boldsymbol{\%}$ Acum. & Firmas & \% & \% Acum. \\
\hline 1 & 7 & 3,28 & 3,28 & 7 & 0,54 & 0,54 \\
\hline 2 & 25 & 11,74 & 15,02 & 50 & 3,87 & 4,41 \\
\hline 3 & 23 & 10,8 & 25,82 & 69 & 5,34 & 9,75 \\
\hline 4 & 28 & 13,15 & 38,97 & 112 & 8,68 & 18,43 \\
\hline 5 & 39 & 18,31 & 57,28 & 195 & 15,1 & 33,53 \\
\hline 7 & 22 & 10,33 & 67,61 & 132 & 10,22 & 43,75 \\
\hline 8 & 23 & 10,8 & 78,41 & 161 & 12,47 & 56,22 \\
\hline 10 & 13 & 6,11 & 84,52 & 104 & 8,06 & 64,28 \\
\hline 11 & 6 & 2,82 & 87,34 & 54 & 4,18 & 68,46 \\
\hline 12 & 3 & 1,4 & 88,74 & 30 & 2,33 & 70,79 \\
\hline 13 & 3 & 1,4 & 90,14 & 33 & 2,56 & 73,35 \\
\hline 14 & 2 & 1,4 & 91,54 & 36 & 2,79 & 76,14 \\
\hline 16 & 3 & 0,94 & 92,48 & 26 & 2,01 & 78,15 \\
\hline 17 & 4 & 1,41 & 93,89 & 42 & 3,25 & 81,4 \\
\hline 18 & 4 & 1,88 & 95,77 & 60 & 4,65 & 86,05 \\
\hline 22 & 1 & 1,88 & 97,65 & 64 & 4,96 & 91,01 \\
\hline 30 & 1 & 0,47 & 98,12 & 17 & 1,32 & 92,33 \\
\hline & 1 & 0,47 & 98,59 & 18 & 1,39 & 93,72 \\
\hline & 1 & 0,47 & 99,53 & 29 & 2,25 & 97,67 \\
\hline & 1 & 0,47 & 100 & 30 & 2,33 & 100 \\
\hline & 21 & & & 1291 & & \\
\hline
\end{tabular}

Fuente: Elaboración propia.

Sin embargo, si se unifican los 173 registros que coinciden en ambas bases de datos y a estos se añaden las 411 publicaciones que están sólo en Scopus más las 40 que únicamente se encuentran en WoS se obtiene un total de 624 trabajos y 3024 firmas. De esta forma, al analizar de forma general el índice de coautoría este es igual a 4.85 (tabla 3).

Tabla 3. Cantidad de firmas, número de trabajos, y cálculo del índice de coautoría en general.

\begin{tabular}{|c|c|c|c|c|c|c|}
\hline Firmas & Trabajos & $\%$ & \% Acum. & Firmas & $\%$ & \% Acum. \\
\hline 1 & 50 & 8,01 & 8,01 & 50 & 1,65 & 1,65 \\
\hline 2 & 71 & 11,38 & 19,39 & 142 & 4,7 & 6,35 \\
\hline 3 & 90 & 14,43 & 33,82 & 270 & 8,93 & 15,28 \\
\hline 4 & 126 & 20,2 & 54,02 & 504 & 16,66 & 31,94 \\
\hline 5 & 121 & 19,39 & 73,41 & 605 & 20,01 & 51,95 \\
\hline 6 & 58 & 9,29 & 82,7 & 348 & 11,51 & 63,46 \\
\hline 7 & 35 & 5,61 & 88,31 & 245 & 8,1 & 71,56 \\
\hline 8 & 21 & 3,37 & 91,68 & 168 & 5,55 & 77,11 \\
\hline 9 & 13 & 2,08 & 93,76 & 117 & 3,87 & 80,98 \\
\hline 10 & 6 & 0,96 & 94,72 & 60 & 1,99 & 82,97 \\
\hline 11 & 5 & 0,8 & 95,52 & 55 & 1,82 & 84,79 \\
\hline 12 & 6 & 0,96 & 96,48 & 72 & 2,38 & 87,17 \\
\hline 13 & 3 & 0,48 & 96,96 & 39 & 1,29 & 88,46 \\
\hline 14 & 3 & 0,48 & 97,44 & 42 & 1,39 & 89,85 \\
\hline 15 & 5 & 0,8 & 98,24 & 75 & 2,48 & 92,33 \\
\hline 16 & 5 & 0,8 & 99,04 & 90 & 2,98 & 95,31 \\
\hline 17 & 1 & 0,16 & 99,2 & 17 & 0,56 & 95,87 \\
\hline 18 & 1 & 0,16 & 99,36 & 18 & 0,59 & 96,46 \\
\hline 22 & 1 & 0,16 & 99,52 & 22 & 0,73 & 97,19 \\
\hline 26 & 1 & 0,16 & 99,68 & 26 & 0,86 & 98,05 \\
\hline 29 & 1 & 0,16 & 99,84 & 29 & 0,96 & 99,01 \\
\hline \multirow[t]{3}{*}{30} & 1 & 0,16 & 100 & 30 & 0,99 & 100 \\
\hline & 624 & & & 3024 & & \\
\hline & & & Índice: 4,85 & & & \\
\hline
\end{tabular}

Fuente: Elaboración propia. 
La figura 2 manifiesta como el índice de coautoría ha tenido pequeños incrementos por año en el período analizado. Esto indica una propensión al trabajo en cooperación por parte de los autores.

Figura 2. Evolución del índice de coautoría.

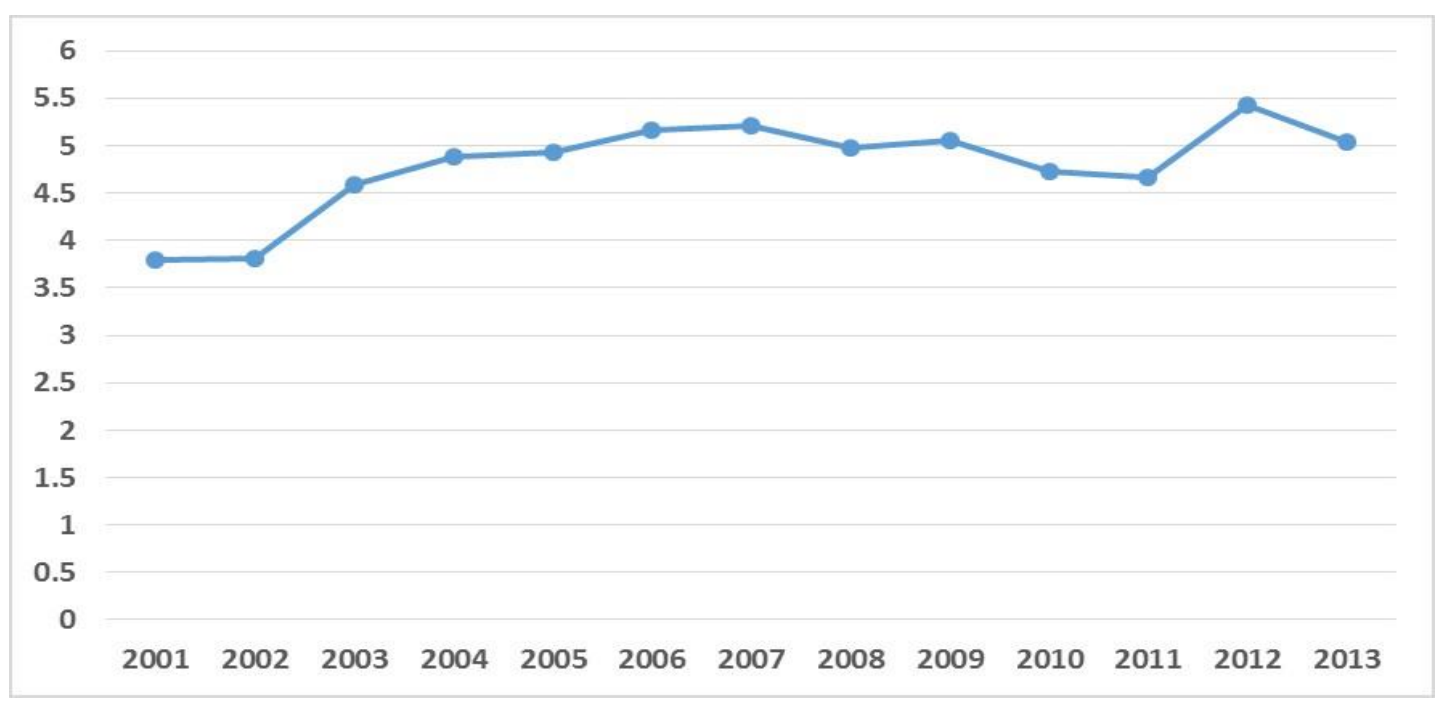

Fuente: Elaboración propia.

A pesar de ello no se detectó la existencia de hiperautoría. El rango de autores firmantes preponderante se encuentra entre 4 y 5 autores, y el mayor número de autores detectado fue de 30 autores en un artículo (figura 3).

Figura 3. Rango de autores firmantes por documentos.

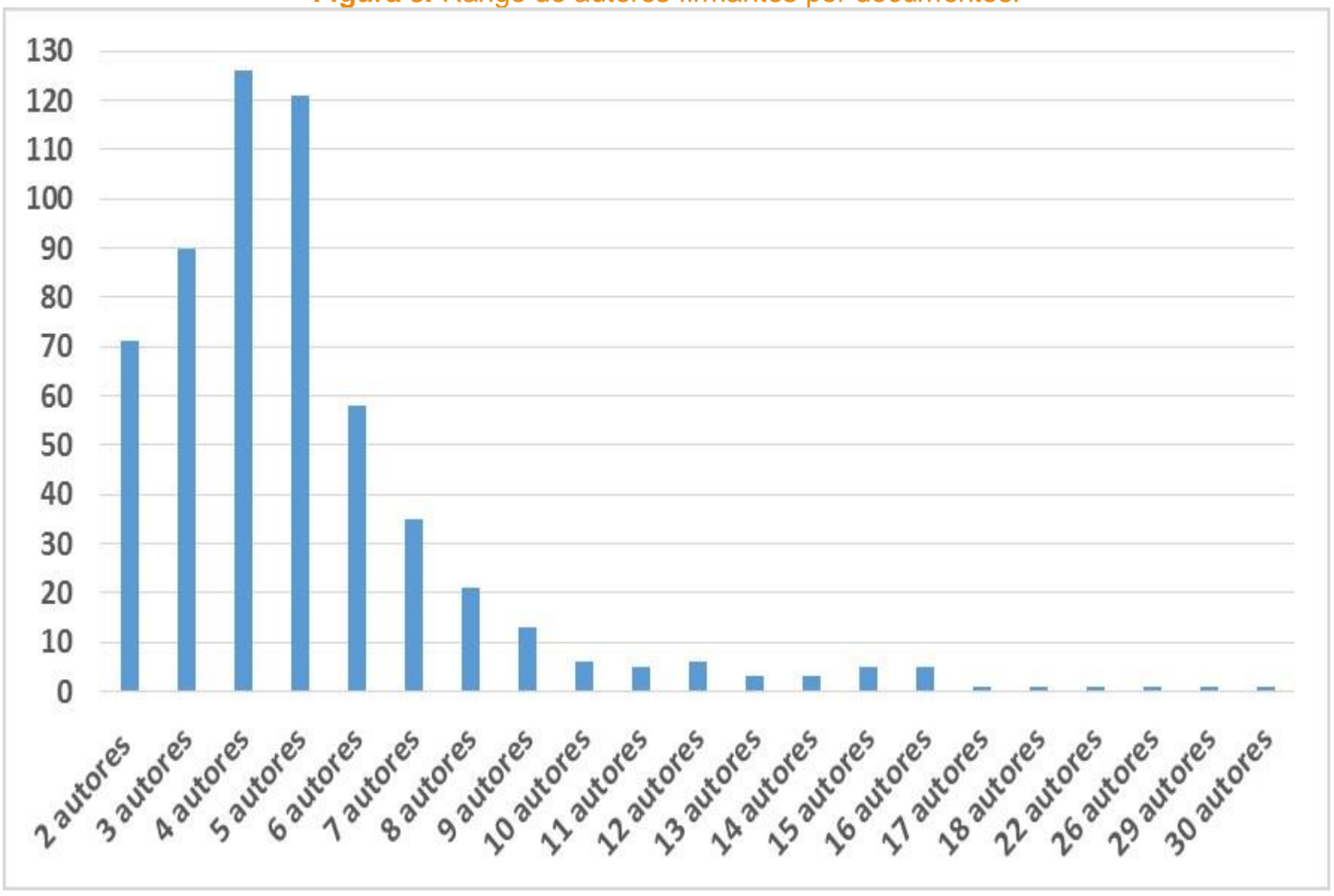

Fuente: Elaboración propia. 
Para comprobar si existe relación entre el impacto científico de las publicaciones y el número de autores se analizaron las citaciones con respecto al rango de autores firmantes. Las tablas 4 y 5 muestran el estudio por separado de las dos bases de datos, dadas las diferencias existentes entre Scopus y WoS en el total de documentos registrados, así como en la cantidad de citas que han recibido los documentos.

El artículo firmado por 30 autores fue el que mayor cantidad de citas recibió en ambas bases de datos, en tanto el promedio de citas de los documentos firmados por un único autor no llega a 1.00. Aunque hubo un artículo firmado por 29 autores (tabla 5) y otro firmado por 18 autores (tabla 4) que no recibieron citas, la generalidad demuestra que las publicaciones en coautoría tienen mayor porciento de documentos citados.

La coautoría en las publicaciones analizadas evidencia la existencia de distintos tipos de colaboración. En la figura 4 se observa el predominio de la colaboración intrainstitucional, presente en más de 200 documentos. Otro tipo de colaboración que predomina es la colaboración a nivel nacional, dentro de esta se destaca el trabajo conjunto entre autores de instituciones del propio territorio, el cual constituye el 55\% de este tipo de cooperación. En el caso de la cooperación con autores de otros países se aprecia la presencia de colaboración exclusivamente internacional, y también la colaboración internacional-nacional. Sólo 50 documentos firmados por un único autor no muestran colaboración.

Tabla 4. Análisis de citaciones en relación con el rango de autores firmantes en Scopus.

\begin{tabular}{|c|c|c|c|c|c|}
\hline Autores firmantes & Documentos (Doc) & Doc citados & $\%$ Doc citados & Citas recibidas & Promedio citas / Doc \\
\hline 1 & 47 & 10 & $21 \%$ & 25 & 0.53 \\
\hline 2 & 70 & 24 & $34 \%$ & 182 & 2.60 \\
\hline 3 & 88 & 29 & $33 \%$ & 101 & 1.15 \\
\hline 4 & 123 & 29 & $24 \%$ & 150 & 1.22 \\
\hline 5 & 115 & 38 & $33 \%$ & 230 & 2.00 \\
\hline 6 & 55 & 20 & $36 \%$ & 131 & 2.38 \\
\hline 7 & 32 & 20 & $62 \%$ & 139 & 4.34 \\
\hline 8 & 15 & 9 & $60 \%$ & 128 & 8.53 \\
\hline 9 & 10 & 5 & $50 \%$ & 90 & 9.00 \\
\hline 10 & 5 & 3 & $60 \%$ & 7 & 1.40 \\
\hline 11 & 4 & 3 & $75 \%$ & 5 & 7.25 \\
\hline 12 & 6 & 5 & $83 \%$ & 47 & 3.50 \\
\hline 13 & 2 & 1 & $50 \%$ & 7 & 14.00 \\
\hline 14 & 1 & 1 & $100 \%$ & 14 & 29.50 \\
\hline 15 & 4 & 3 & $75 \%$ & 118 & 1.00 \\
\hline 16 & 2 & 2 & $100 \%$ & 2 & 2.00 \\
\hline 17 & 1 & 1 & $100 \%$ & 2 & 0 \\
\hline 18 & 1 & 0 & $0 \%$ & 0 & 10.00 \\
\hline 22 & 1 & 1 & $100 \%$ & 10 & 6.00 \\
\hline 26 & 1 & $100 \%$ & 6 & 201 & \\
\hline 30 & 1 & $100 \%$ & & \\
\hline
\end{tabular}

Fuente: Elaboración propia.

La evolución de la colaboración por años muestra que los documentos sin colaboración presentan una tendencia a disminuir. También ha disminuido en los últimos años la colaboración intrainstitucional que del 2001 al 2007 tuvo los valores más elevados. En tanto la cooperación exclusivamente internacional, así como la de carácter internacional-nacional han presentado una ligera predisposición al aumento en los últimos años. Un incremento significativo presentó la colaboración nacional en el año 2012 (figura 5). 
Tabla 5. Análisis de citaciones en relación con el rango de autores firmantes en WoS.

\begin{tabular}{|c|c|c|c|c|c|}
\hline Autores firmantes & Documentos (Doc) & Doc citados & $\%$ Doc citados & Citas recibidas & Promedio Citas / Doc \\
\hline 1 & 7 & 3 & $43 \%$ & 4 & 0.57 \\
\hline 2 & 25 & 18 & $72 \%$ & 180 & 7.20 \\
\hline 3 & 23 & 11 & $48 \%$ & 37 & 1.61 \\
\hline 4 & 28 & 11 & $39 \%$ & 99 & 3.54 \\
\hline 5 & 39 & 21 & $54 \%$ & 226 & 5.79 \\
\hline 6 & 22 & 14 & $64 \%$ & 121 & 5.50 \\
\hline 7 & 23 & 13 & $57 \%$ & 127 & 5.52 \\
\hline 8 & 13 & 6 & $46 \%$ & 91 & 7.00 \\
\hline 9 & 6 & 2 & $33 \%$ & 77 & 12.83 \\
\hline 10 & 3 & 1 & $33 \%$ & 1 & 0.33 \\
\hline 11 & 3 & 2 & $66 \%$ & 2 & 0.66 \\
\hline 12 & 3 & 3 & $100 \%$ & 32 & 10.66 \\
\hline 13 & 2 & 1 & $50 \%$ & 7 & 3.50 \\
\hline 14 & 3 & 1 & $33 \%$ & 10 & 3.33 \\
\hline 15 & 4 & 3 & $75 \%$ & 85 & 21.25 \\
\hline 16 & 4 & 3 & $75 \%$ & 44 & 11.00 \\
\hline 17 & 1 & 1 & $100 \%$ & 3 & 3.00 \\
\hline 18 & 1 & 0 & $0 \%$ & 0 & 0 \\
\hline 22 & 1 & 1 & $100 \%$ & 7 & 7.00 \\
\hline 29 & 1 & 0 & $0 \%$ & 0 & 0 \\
\hline 30 & 1 & 1 & $100 \%$ & 189 & 189.00 \\
\hline
\end{tabular}

Fuente: Elaboración propia.

Figura 4. Documentos según el tipo de colaboración.

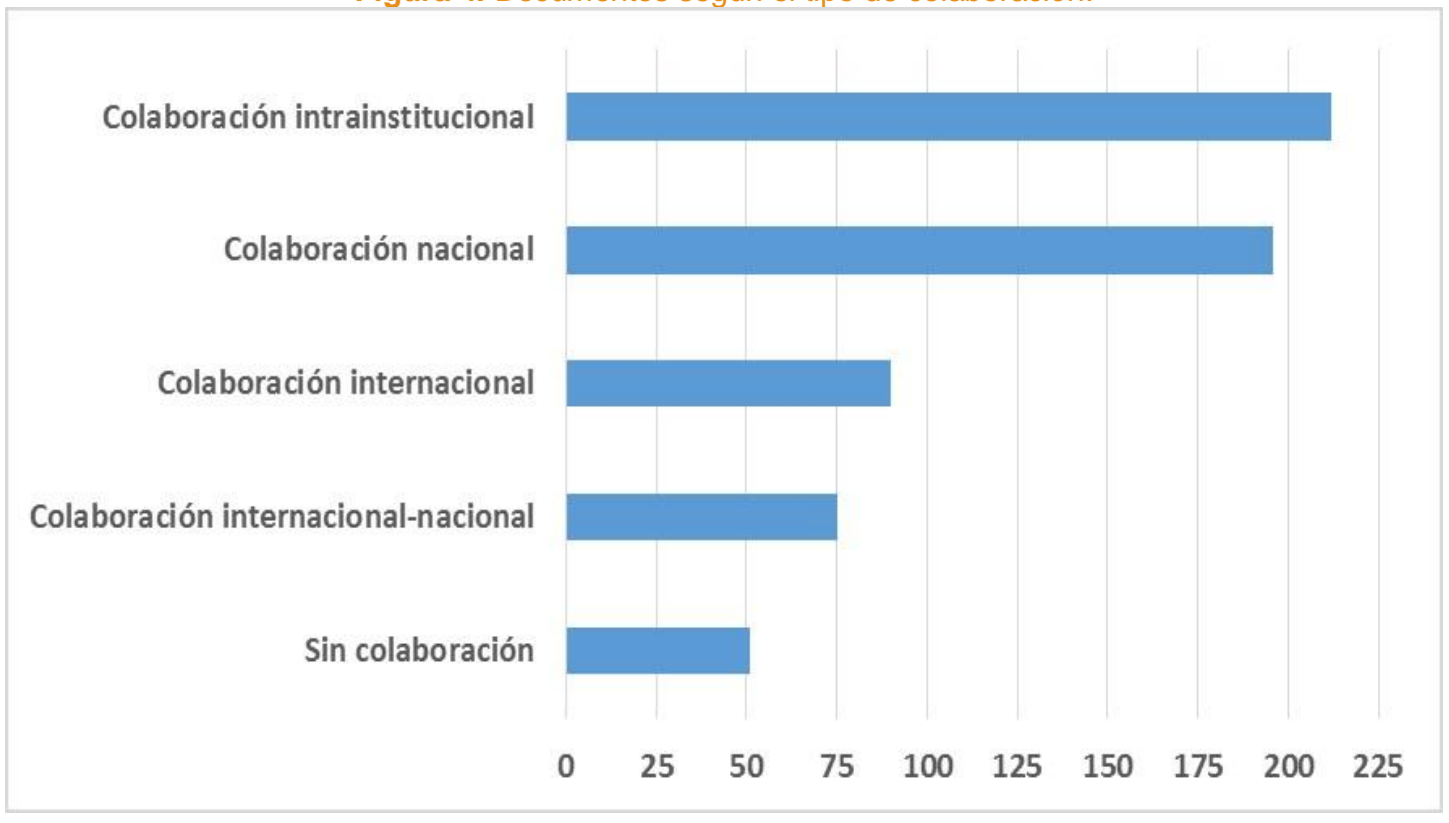

Fuente: Elaboración propia. 
Figura 5. Evolución de la colaboración por años.

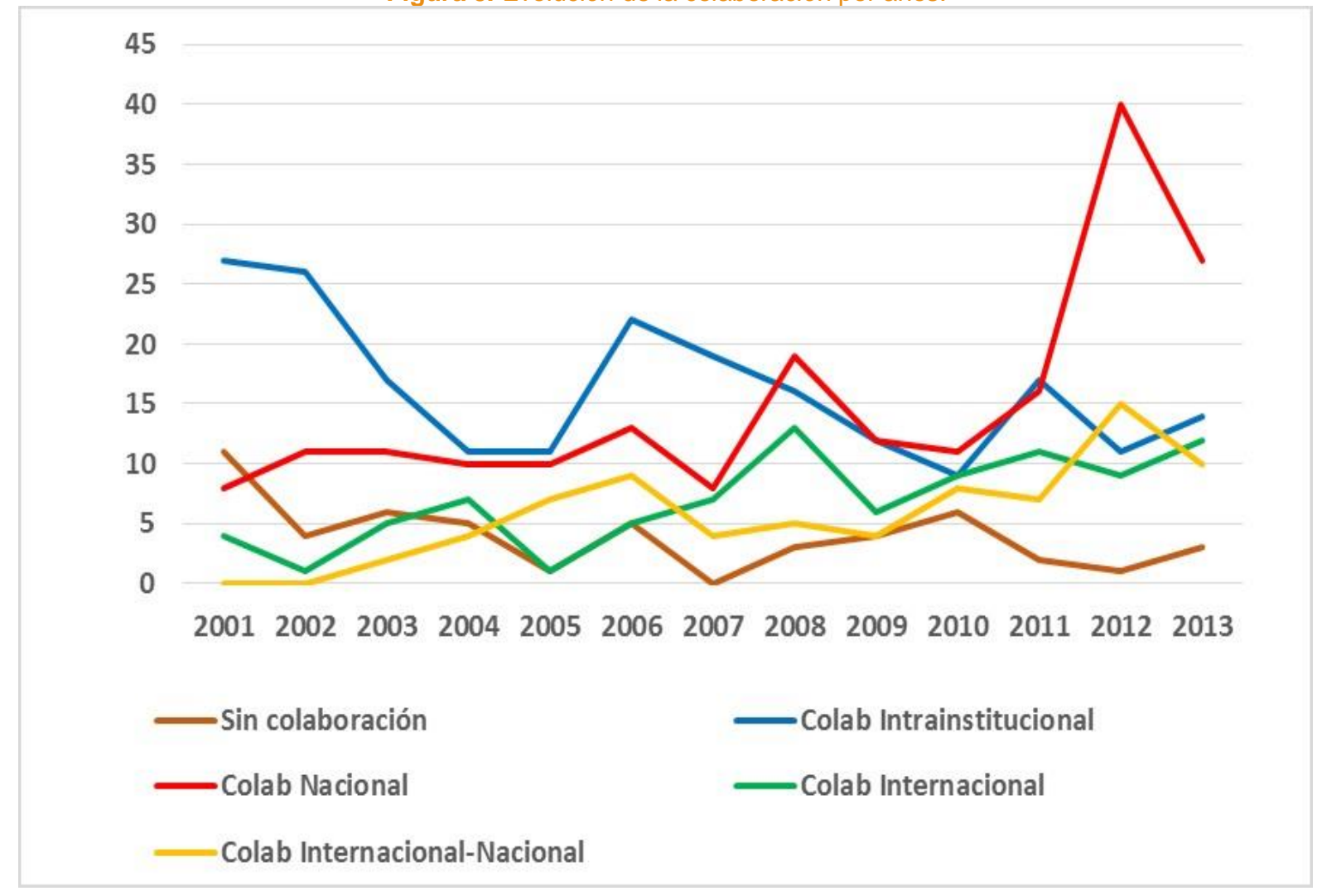

Fuente: Elaboración propia.

Figura 6. Colaboración entre instituciones camagüeyanas e instituciones cubanas

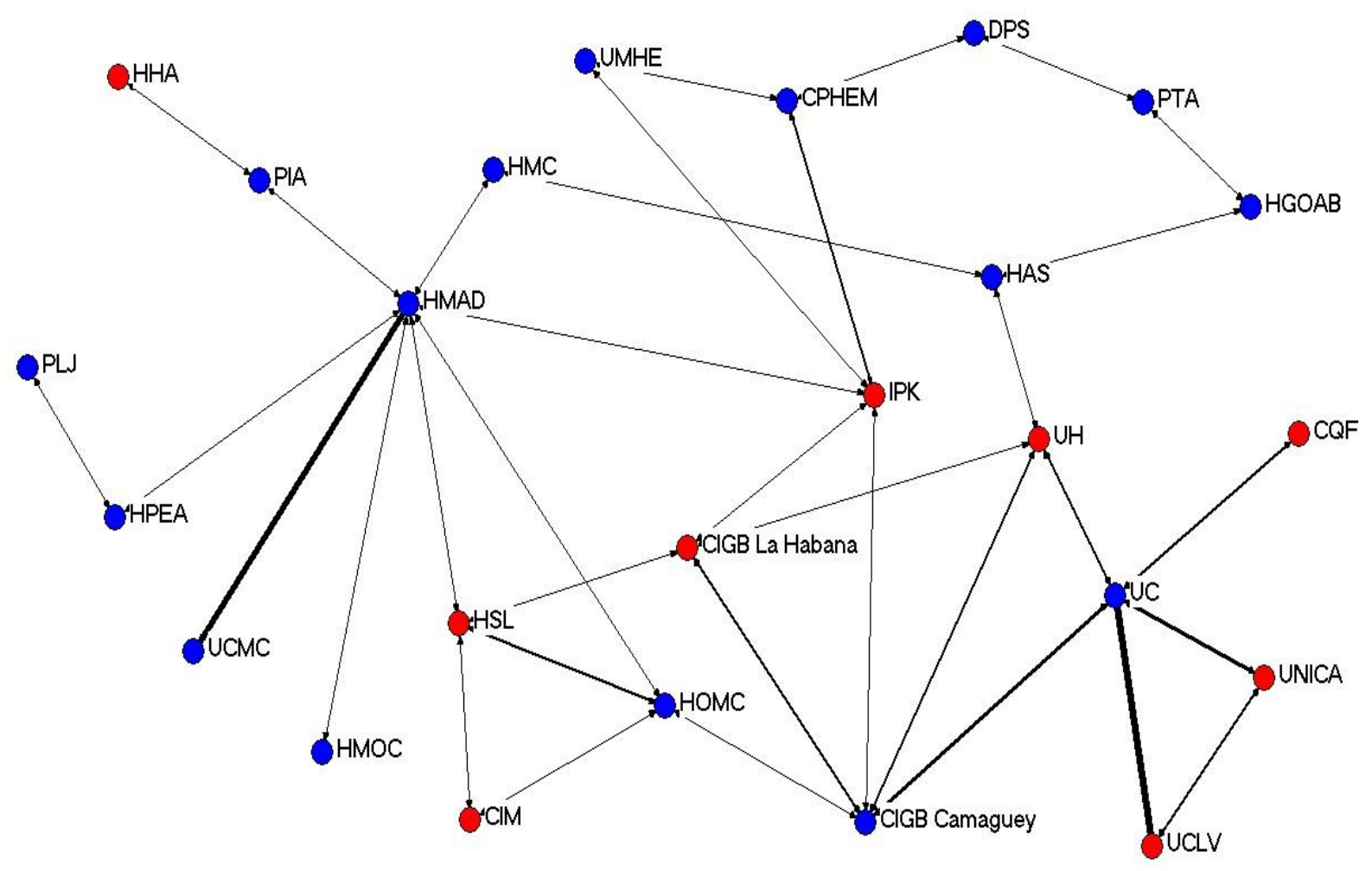

Fuente: Elaboración propia. 
Para el análisis de redes de colaboración institucional se estudiaron los documentos en la categoría colaboración nacional y también en la internacional. En la figura 6 se muestran las instituciones nacionales cuyos autores cooperaron 5 o más veces. Se señalan en color azul las entidades camagüeyanas y en color rojo aquellas que son de otras provincias cubanas (ver anexo para las siglas que identifican las instituciones). Las relaciones más fuertes se han establecido entre la Universidad de Camagüey y la Universidad Central de Las Villas, lo que se evidencia en más de 35 publicaciones. Una de las principales causas de que estos vínculos se hayan fortalecido es la superación de profesores de la Universidad de Camagüey en la Universidad Central de Las Villas, evidente en el desarrollo de doctorados, maestrías y cursos de posgrado. Asimismo, se aprecia una fuerte relación entre el Hospital Manuel Ascunce Domenech y la Universidad de Ciencias Médicas de Camagüey, evidente en más de 30 documentos. En este caso la unión está dada por el trabajo del hospital como unidad docente, en la cual profesores y estudiantes de la Universidad de Ciencias Médicas no sólo llevan a cabo sus actividades prácticas, sino que también investigan en conjunto con los especialistas del centro.

La figura 7 representa las instituciones internacionales y camagüeyanas cuyos autores cooperaron 5 o más veces. Se señalan en color azul las entidades de la provincia Camagüey y en color rojo aquellas que son de otros países. Los vínculos más fuertes se han establecido entre la Universidad de Camagüey y la Universidad de Santiago de Compostela (España), quienes han compartido la autoría de más de 10 documentos. Estos lazos se han desarrollado por el trabajo continuado de colegas de ambas universidades en investigación sobre estructura molecular y descriptores moleculares. También, existen relaciones estrechas entre el Hospital Manuel Ascunce Domenech y el Departamento de Emergencias de Los Llanos de Aridane (Canarias, España), cuyos autores han generado más de 10 publicaciones. Esta relación ha estado dada por la frecuente cooperación entre especialistas en urología de ambos centros. Llama la atención el trabajo reiterado de un grupo integrado por un autor del Policlínico de Previsora con autores de entidades de distintos países, principalmente de la Unidad Médica Integral Alergología y Pediatría de Tehuacán (México). Este grupo ha desarrollado una amplia labor investigativa sobre agentes causales de alergias, así como la inmunoterapia adecuada para distintos casos de alergia y de asma.

Figura 7. Colaboración entre instituciones camagüeyanas e instituciones internacionales.
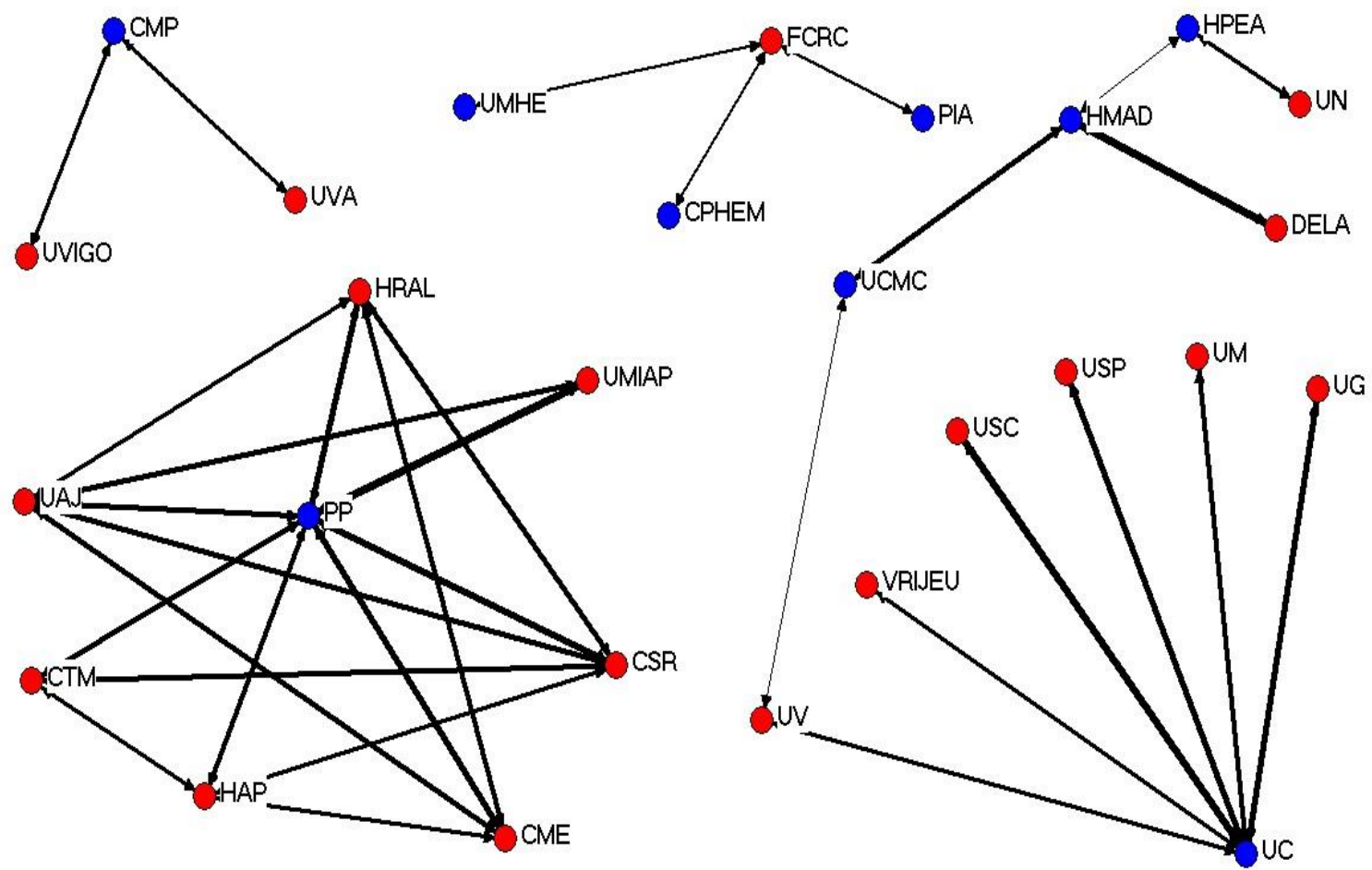

Fuente: Elaboración propia. 
El volumen de documentos firmados en coautoría hace que se formen diversas redes de investigadores. Se tomó como objeto de análisis aquellas redes que se forman a partir del trabajo de los autores más productivos y sus colaboradores. Estas redes son las que mayor cantidad de investigadores involucran en cada sector estudiado, por lo que constituyen las más representativas.

En las figuras siguientes se representan las redes de colaboración identificadas en los diferentes sectores. Los nodos en color azul representan los autores con mayor grado de centralidad en la red y el grosor de las líneas indica la intensidad de las relaciones entre los miembros.

En el caso del sector de la salud, la figura 8 muestra una red conformada por 65 investigadores, donde los autores con mayor grado de centralidad son Pila-Pérez $\mathrm{R}$ y Pila-Peláez R, estos autores son los más productivos con 52 y 49 publicaciones respectivamente. Los vínculos más fuertes se establecieron entre estos propios investigadores, los cuales cooperaron en la elaboración de 48 artículos. A su vez, ambos trabajaron más de 20 veces con Holguín V y Rosales P. Estos cuatro autores trabajan en el Servicio de Medicina Interna del Hospital Manuel Ascunce Domenech.

En la Universidad de Camagüey, la autora más productiva es Caballero Y, quien participa en la autoría de 31 documentos y posee el mayor grado de centralidad de la red integrada por 40 investigadores (figura 9). La mayor cantidad de colaboraciones se han establecido entre esta autora y Bello R, investigador de la Universidad Central de las Villas (UCLV), con quien ha trabajado en 24 publicaciones. También ha existido, aunque en menor medida un trabajo reiterado de cooperación con García MM y Arco L, de la UCLV, y con Filiberto Y de la propia Universidad de Camagüey. Todos estos autores trabajan e investigan en el área de Ciencias de la Computación y/o Ingeniería Informática.

El Centro Meteorológico Provincial constituye la entidad más fructífera dentro de las instituciones que pertenecen al CITMA. A este centro pertenece Antuña JC, autor más productivo con 22 publicaciones, el cual tiene el mayor grado de centralidad en la red constituida por 40 investigadores (figura 10). Los vínculos de cooperación más estrechos se han establecido entre este autor, Barja B y Estevan R, en la firma de 12 y 9 publicaciones respectivamente. Estos autores trabajan en el Grupo de Óptica Atmosférica del Centro Meteorológico.

Figura 8. Colaboración entre investigadores del Servicio de Medicina Interna del Hospital Manuel Ascunce Domenech y otros autores.

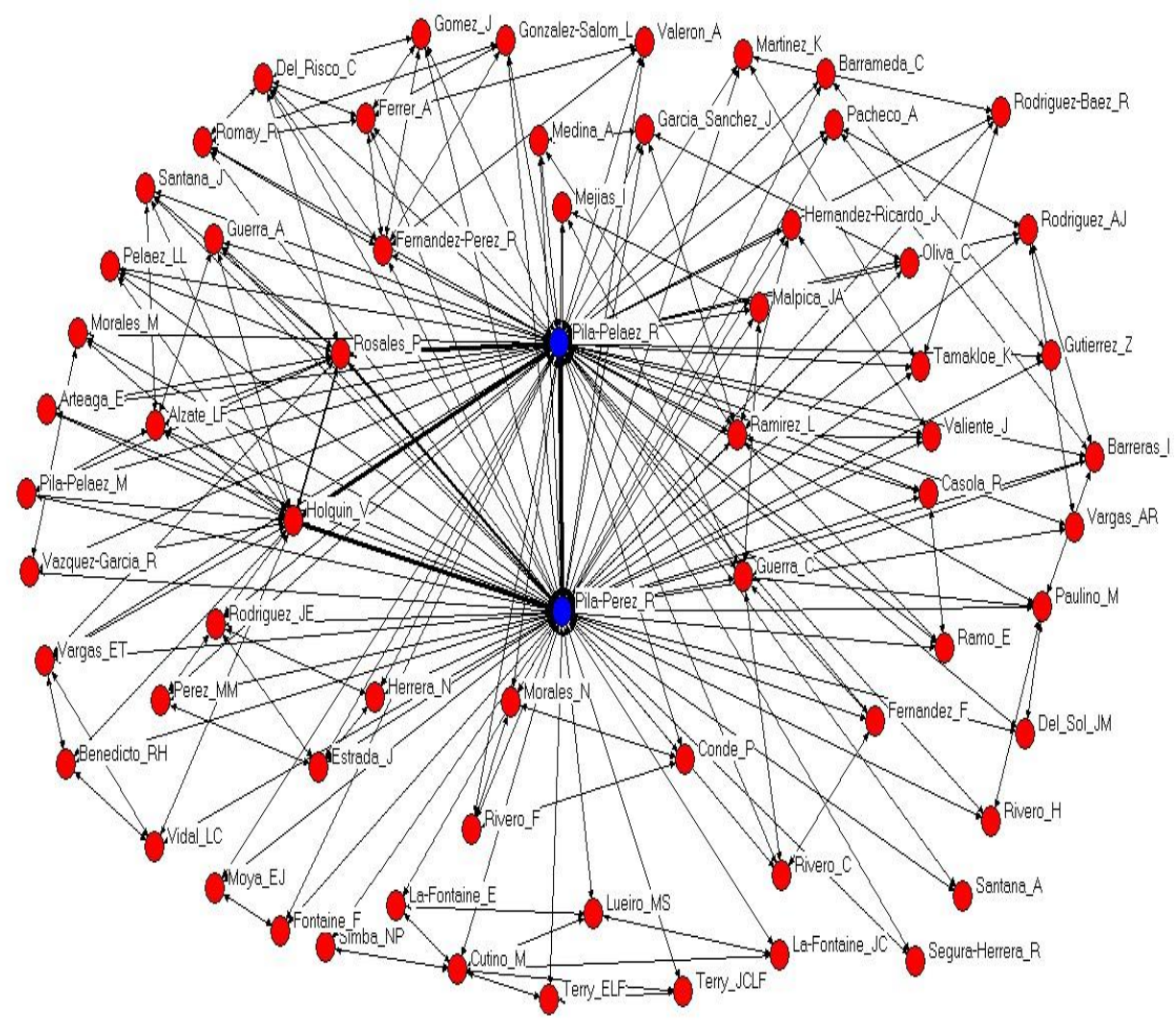

Fuente: Elaboración propia. 
Figura 9. Colaboración entre investigadores de la Facultad de Informática de la Universidad de Camagüey y otros autores.

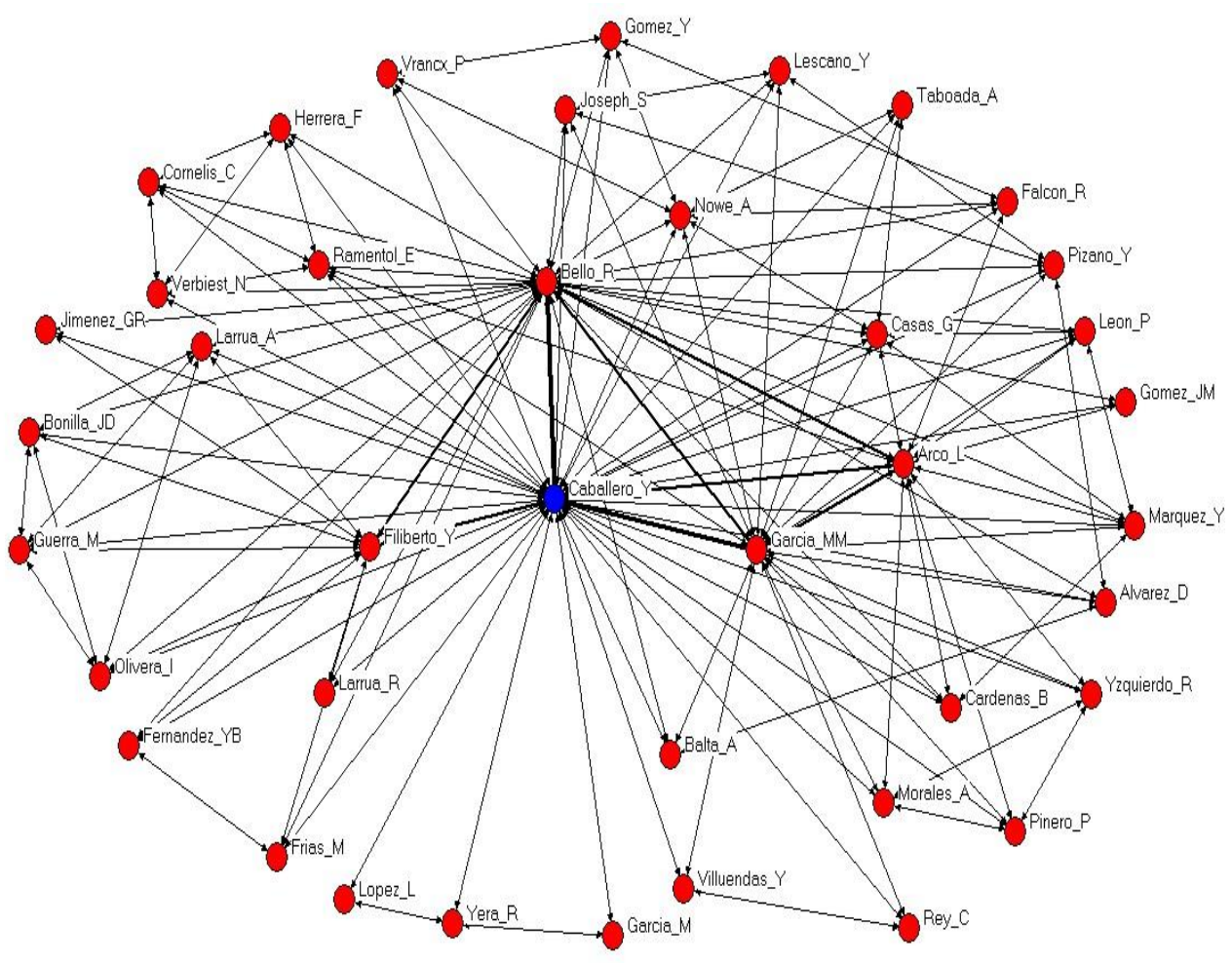

Fuente: Elaboración propia.

Figura 10. Colaboración entre investigadores del Grupo de Óptica Atmosférica del Centro Meteorológico Provincial de Camagüey y otros autores.

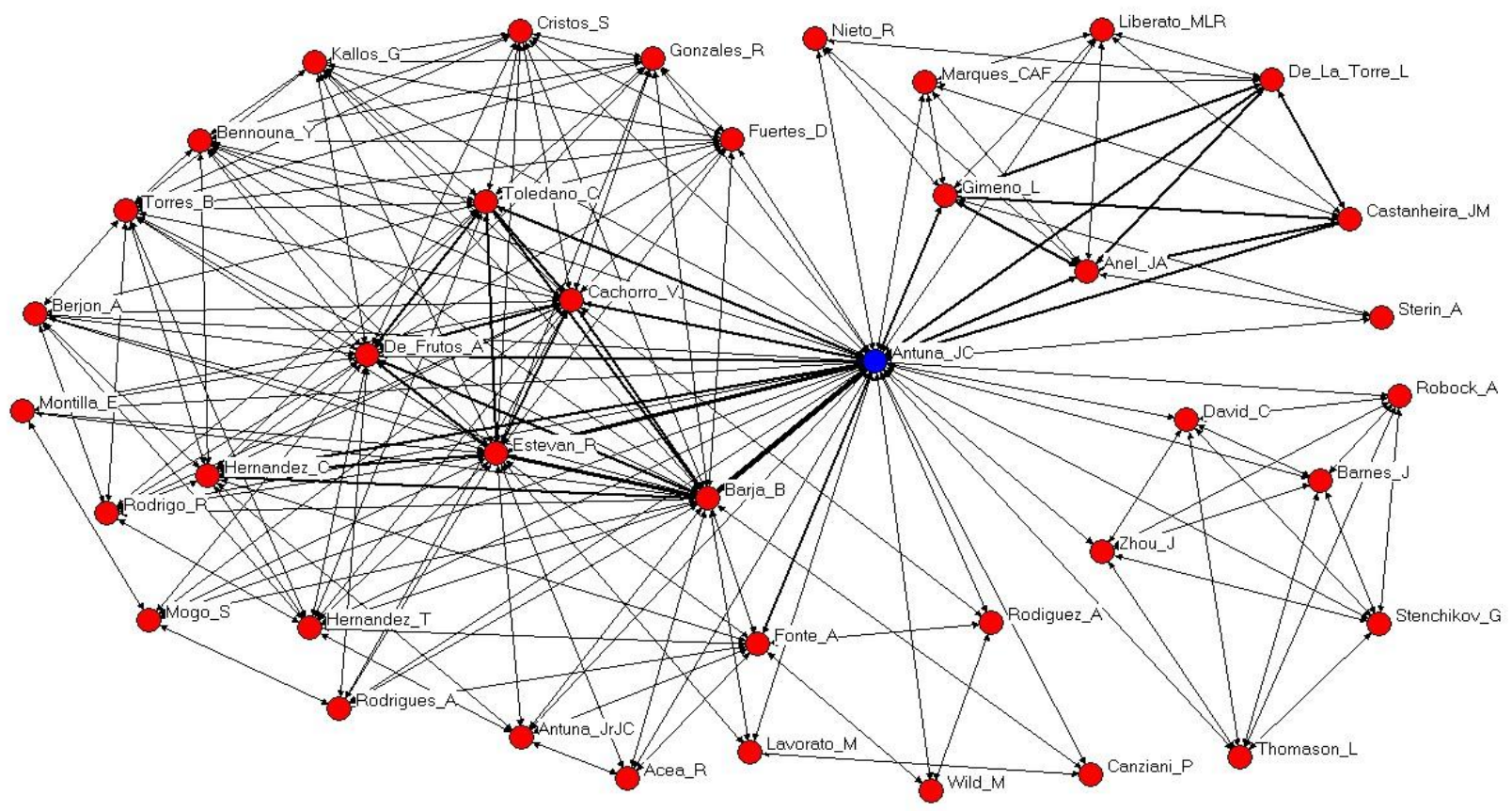

Fuente: Elaboración propia.

La red representativa del CIGB está formada por 37 investigadores y presenta 6 autores con un alto grado de centralidad. De ellos, Baker RB participa en la autoría de 13 publicaciones, trabajando con Junco JA, Fuentes F y Calzada $L$ en 11, y con Bover E en 10. Todos estos autores pertenecen al propio CIGB de Camagüey. No obstante, se aprecia también cooperación con investigadores que trabajan en el CIGB de La Habana, como Reyes $\mathrm{O}$ y Guillen $\mathrm{G}$, quienes participan en la elaboración de 7 artículos (figura 11). 


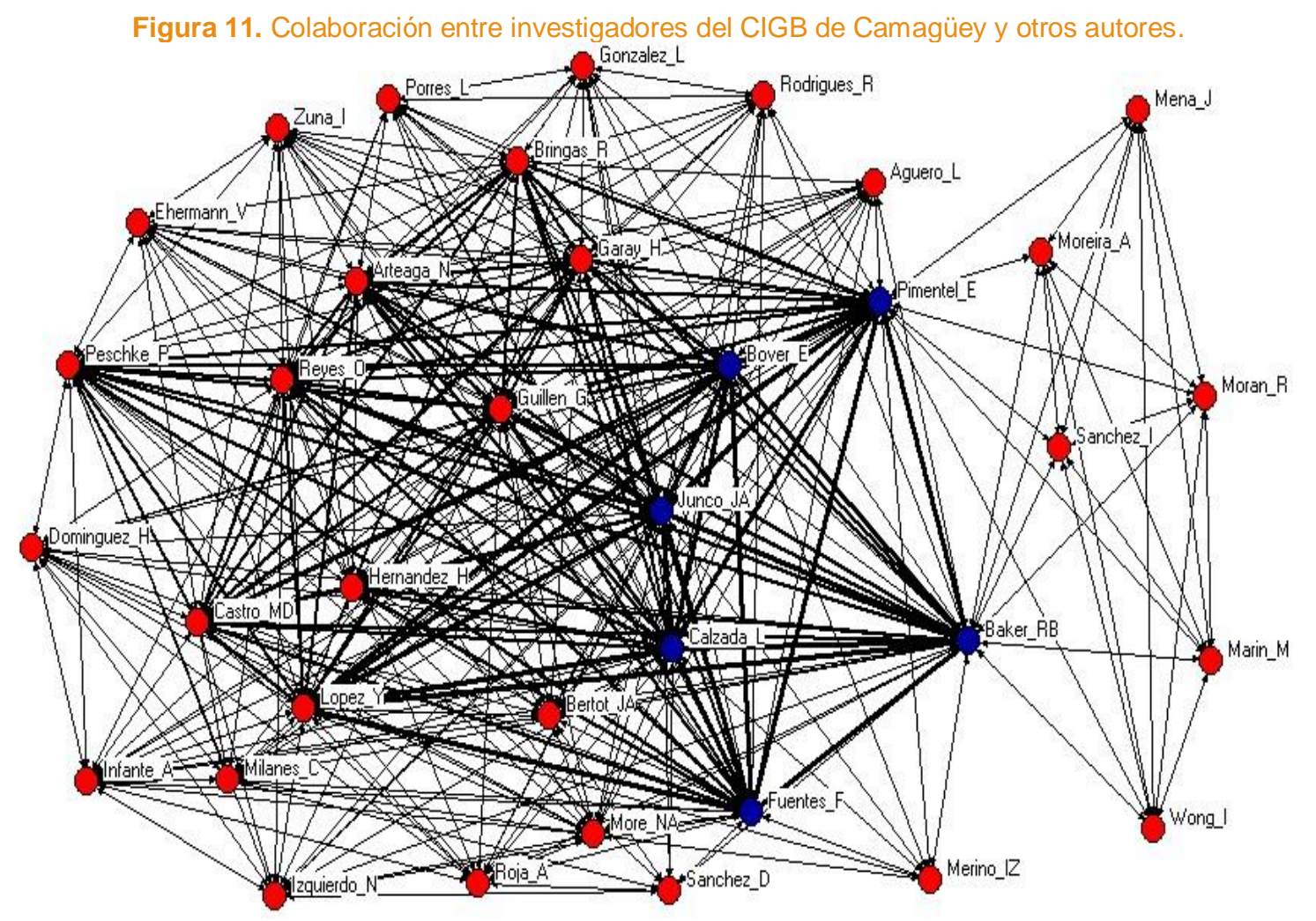

Fuente: Elaboración propia.

\section{Conclusiones}

La coautoría está presente en un 92\% de la producción científica camagüeyana. En todos los sectores se aprecia la firma de publicaciones entre 2 o más autores. El número medio de autores firmantes, acorde con el índice de coautoría, oscila entre 4 y 5 autores. El mayor rango de autores firmantes es 30 en un artículo.

No abundan los documentos con más de 15 autores, a pesar de que el análisis de citaciones en ambas bases de datos mostró que, si bien existen excepciones, el promedio de citas por documentos suele ser más alto en las publicaciones con mayor número de investigadores. Esta cuestión había sido estudiada por Hsu y Huang (2011), quienes determinaron que los artículos firmados por un solo autor acumulan menor frecuencia de citas, mientras que los artículos elaborados por un número alto de co-autores reciben un número de citas promedio superior.

Se observa el predominio de la colaboración intrainstitucional en el período. El análisis de las redes que se forman a partir del trabajo de los autores más productivos evidencia la primacía de este tipo de colaboración. Los vínculos colaborativos más fuertes se han establecido entre autores de una misma institución, excepto en el caso de la Universidad de Camagüey, donde se ha trabajado estrechamente con investigadores de la UCLV. Sin embargo, a partir del año 2008 se observa una sutil tendencia a que este tipo de cooperación disminuya y aumenten los otros tipos de trabajo colaborativo, principalmente la colaboración nacional.

En el análisis de la colaboración institucional se destacan la Universidad de Camagüey y el Hospital Manuel Ascunce Domenech. Estas instituciones son las que han establecido vínculos colaborativos más fuertes con otras entidades, tanto cubanas como extranjeras.

La internacionalización de la producción científica fue muy baja hasta el año 2005. A partir del 2006, tanto la colaboración internacional como la colaboración internacional-nacional han tenido un ligero incremento, pero continúan por debajo de la colaboración nacional e intrainstitucional. Aún deben desarrollarse voluntades en los autores camagüeyanos para trabajar con investigadores de otros países. 


\section{Referencias}

Franceschet, M.; Costantini, A. (2010). The effect of scholar collaboration on impact and quality of academic papers. Journal of Informetrics, vol. 4 (4), 540-553.

Gavel, Y.; Iselid, L. (2008). Web of Science and Scopus: a journal title overlap study, Online Information Review, vol. 32 (1), 8 21

Gazni, A.; Didegah, F. (2011). Investigating different types of research collaboration and citation impact: a case study of Harvard University's publications. Scientometrics, vol. 87, 251-265. http://doi:10.1007/s11192-011-0343-8

Glänzel, W. (2002). Coauthorships patterns and trends in the Sciences (1980-1998): A bibliometric study with implications for database indexing and search strategies. Library Trends, vol. 50 (3), 461-473.

Glänzel, W.; Schubert, A. (2004). Analysing scientific networks through co-authorship. En: Moed, H.; Glänzel, W.; Schmoch, U. (editores) Handbook of Quantitative Science and Technology Research. Statistics in Studies of S\&T Systems. Kluwer Academic Publishers, Netherlands.

González-Alcaide, G.; Gómez, J. (2014). La colaboración científica: principales líneas de investigación y retos de futuro. Revista Española de Documentación Científica, vol. 37 (4). http://dx.doi.org/10.3989/redc.2014.4.1186

Hsu, J.; Huang, D. (2011). Correlation between impact and collaboration, Scientometrics, vol. 86 (2), 317-324.

Leydesdorff, L.; Wagner, C. (2009). Is the United States losing ground in Science?: a global perspective on the world science system. Scientometrics, vol. 78 (1), 23-36.

Olmeda-Gómez, C.; Perianes-Rodríguez, A.; Ovalle-Perandones, M. A. (2008). Estructura de las redes de colaboración científica de las universidades españolas. Ibersid, vol. 2, 129-140.

Osca-Lluch, J. (2010). Aplicación del análisis de redes al estudio de la investigación española de historia de la ciencia. Redes. Revista Hispana para el análisis de redes sociales, vol. 19 (6). Disponible en http://revista-redes.rediris.es

Padial, A.A.; Nabout, J.C.; Siqueira, T.; Felizola, J.A. (2010). Weak evidence for determinants of citation frequency in ecological articles. Scientometrics, vol. 85 (1), 1-12.

Russell, M.; Ainsworth, S.; Narváez-Berthelemot, N. (2006). Colaboración científica de la Universidad Nacional Autónoma de México (UNAM) y su política institucional. Revista Española de Documentación Científica, vol. 29 (1), 56-73.

Russell, J.; Madera, M.J.; Ainsworth, S. (2009). El análisis de redes en el estudio de la colaboración científica. Redes. Revista Hispana para el análisis de redes sociales, vol. 17 (2), 39-47. Disponible en http://revista-redes.rediris.es

Sancho, R., Morillo, F., De Filippo, D., Gómez, I. \& Fernández, M. (2006). Indicadores de colaboración científica inter-centros en los países de América Latina. Interciencia, vol. 31 (4), 284-292.

Souza, S.A.; Chittó, I.R. (2010). Colaboração científica: revisão teórico-conceitual. Perspectivas em Ciência da Informação, vol. $15(2), 42-55$

Vieira, E.; Gomes, J. (2010). Citations to scientific articles: Its distribution and dependence on the article features. Journal of Informetrics, vol. 4 (1), 1-13. 


\section{Anexo}

\begin{tabular}{|c|c|}
\hline Instituciones nacionales & Siglas \\
\hline Universidad de Camagüey & UC \\
\hline Universidad Ciencias Médicas de Camagüey & UCMC \\
\hline Hospital Manuel Ascunce Domenech (Camagüey) & HMAD \\
\hline Universidad Central de Las Villas (Villa Clara) & UCLV \\
\hline Centro de Ingeniería Genética y Biotecnología de Camagüey & CIGB Camagüey \\
\hline Hospital Pediátrico Eduardo Agramonte (Camagüey) & HPEA \\
\hline Hospital Amalia Simoni (Camagüey) & HAS \\
\hline Hospital Oncológico Marie Curie (Camagüey) & HOMC \\
\hline Centro de Ingeniería Genética y Biotecnología de La Habana & CIGB La Habana \\
\hline Universidad de La Habana & $\mathrm{UH}$ \\
\hline Centro Provincial de Higiene, Epidemiología y Microbiología (Camagüey) & CPHEM \\
\hline Universidad de Ciego de Ávila & UNICA \\
\hline Instituto de Medicina Tropical Pedro Kouri (La Habana) & IPK \\
\hline Unidad Municipal de Higiene y Epidemiología (Camagüey) & UMHE \\
\hline Hospital Gineco-Obstétrico Ana Betancourt (Camagüey) & HGOAB \\
\hline Hospital Militar Octavio de la Concepción (Camagüey) & HMOC \\
\hline Hospital Saturnino Lora (Santiago de Cuba) & HSL \\
\hline Hospital Hermanos Ameijeiras (La Habana) & $\mathrm{HHA}$ \\
\hline Centro de Inmunología Molecular (La Habana) & CIM \\
\hline Policlínico La Jagua Santa Cruz del Sur (Camagüey) & PLJ \\
\hline Dirección Provincial de Salud (Camagüey) & DPS \\
\hline Hospital Martin Chang de Nuevitas (Camagüey) & HMC \\
\hline Policlínico Ignacio Agramonte (Camagüey) & $\mathrm{PIA}$ \\
\hline Policlínico Tula Aguilera (Camagüey) & PTA \\
\hline Centro de Química Farmacéutica (La Habana) & CQF \\
\hline Centro Meteorológico Provincial (Camagüey) & CMP \\
\hline Policlínico Previsora (Camagüey) & $\mathrm{PP}$ \\
\hline Instituciones internacionales & Siglas \\
\hline Universidad Santiago de Compostela (España) & USC \\
\hline Unidad Médica Integral Alergología y Pediatría de Tehuacán (México) & UMIAP \\
\hline Universidad de Granada (España) & UG \\
\hline Universidad de Sao Paulo (Brasil) & USP \\
\hline Centro Médico de Especialidades de Juárez (México) & CME \\
\hline Clínica Santa Rosa El Tigre (Venezuela) & CSR \\
\hline Departamento de Emergencias Los llanos de Aridane Canarias (España) & DELA \\
\hline Universidad de Nottingham (Reino Unido) & UN \\
\hline Fundación Canaria Rafael Clavijo (España) & FCRC \\
\hline Hospital Regional Lic. Adolfo López (México) & HRAL \\
\hline Universidad Autónoma Juárez (México) & UAJ \\
\hline Universidad de Málaga (España) & UM \\
\hline Clínica Torre Médica Ciudad Machala (Ecuador) & CTM \\
\hline Universidad de Vigo (España) & UVIGO \\
\hline Hospital Ángeles Pedregal (México) & HAP \\
\hline Universidad de Valladolid (España) & UVA \\
\hline Vrije Universiteit Bruselas (Bélgica) & VRIJEU \\
\hline Universidad de Valencia (España) & UV \\
\hline
\end{tabular}




\section{Datos de los autores}

\section{Dianelis Olivera Batista}

Master en Bibliotecología y Ciencia de la Información. Profesora Asistente del Departamento de Ciencias de la Información de la Universidad de Camagüey "Ignacio Agramonte Loynaz", Camagüey Cuba.

dianelis.olivera@reduc.edu.cu

\section{María Josefa Peralta González}

Doctora en Documentación e Información Científica. Profesora Titular del Departamento Ciencias de la Información y Vicedecana de Investigación, Internacionalización y Postgrado de la Facultad Matemática, Física y Computación de la Universidad Central "Marta Abreu" de Las Villas, Santa Clara, Cuba.

mjosefa@uclv.edu.cu

\section{Ognara García García}

Doctora en Documentación e Información Científica. Profesora Titular del Departamento Ciencias de la Información y Vicedecana de Formación en la Facultad de Informática y Ciencias Exactas de la Universidad de Camagüey "Ignacio Agramonte Loynaz", Camagüey Cuba.

ognara.garcia@reduc.edu.cu

Recibido - Received: 2017-02-07

Aceptado - Accepted: 2018-01-11

\section{(cc) BY}

This work is licensed under a Creative Commons Attribution 4.0

United States License.

\section{ULLS D-Sorke}

This journal is published by the University Library System of the University of Pittsburgh as part of its D-Scribe Digital Publishing Program and is cosponsored by the University of Pittsburgh Press. 